\title{
Novel biallelic loss-of-function variants in CEP290 cause Joubert syndrome in two siblings
}

\author{
Xiang Wang ${ }^{1}$, Zhu Zhang ${ }^{2,3}$, Xueguang Zhang ${ }^{1}$, Ying Shen ${ }^{1 *}$ and Hongqian Liu ${ }^{2,3^{*}}$ (D)
}

\begin{abstract}
Background: Joubert syndrome (JS) is a rare genetic disorder, which can be defined by brain stem malformation, cerebellar vermis hypoplasia, and consequent "molar tooth sign" (MTS). JS always shares variety of phenotypes in development defects. With the development of next-generation sequencing, dozens of causative genes have been identified to JS so far. Here, we investigated two male siblings with JS and uncovered a novel pathogenesis through combined methods.

Results: The siblings shared similar features of nystagmus, disorders of intellectual development, typical MTS, and abnormal morphology in fourth ventricle. Whole-exome sequencing (WES) and chromosome comparative genomic hybridization $(\mathrm{CGH})$ were then performed on the proband. Strikingly, a maternal inherited nonsense variant (NM_ 025114.3: c.5953G>T [p.E1985*]) in CEP290 gene and a paternal inherited deletion in 12q21.32 including exons 1 to 10 of CEP290 gene were identified in the two affected siblings. We further confirmed the two variants by in vitro experiments: quantitative PCR and PCR sequencing.
\end{abstract}

Conclusions: In this study, we first reported a novel causative mechanism of Joubert syndrome: a copy number variation (CNV) combined with a single-nucleotide variant in CEP290 gene, which can be helpful in the genetic diagnosis of this disease.

Keywords: Joubert syndrome, Copy number variation, Compound heterozygous variants, WES, CGH

\section{Background}

Joubert syndrome (JS) is a genetic ciliopathy disorder with multiple neurologic features of brain malformations, hypotonia, ataxia, and intellectual disability first described in 1969 [1, 2]. With the medical use of magnetic resonance imaging (MRI), JS diagnostic criteria were importantly revised with "molar tooth sign" (MTS), an abnormally deep interpeduncular fossa in midbrain [3-5]. Sharing MTS has

\footnotetext{
* Correspondence: yingcaishen01@163.com; hongqian.liu@163.com 'Department of Obstetrics/Gynecology, Joint Laboratory of Reproductive Medicine (SCU-CUHK), Key Laboratory of Obstetric, Gynecologic and Pediatric Diseases and Birth Defects of Ministry of Education, West China Second University Hospital, Sichuan University, Chengdu 610041, China

${ }^{2}$ Department of Obstetrics and Gynecology, West China Second University Hospital, Sichuan University, Chengdu 610041, China

Full list of author information is available at the end of the article
}

been also used to group any individual displaying additional non-neurological features to Joubert syndrome-related disorders (JSRD) [4]. To date, more than 30 gene variants have been identified in JS, and which allow to group JS into different subtypes (JBTS) [6-8]. Most of these gene variants exhibit autosomal recessive inheritance, and the top three are C5ORF42, CC2D2A, and CEP290 in turn $[9,10]$.

As with other genes associated with JS, CEP290 is a primary cilium-related gene encoding centrosomal protein 290, a large essential protein expressed in almost all tissues and playing a critical role in cell motility and division through effects on centrosome and cilia development [11-13]. Disrupted CEP290 protein (also known as NPHP6) has been found to loss function binding to cellular membranes and microtubules [14], and proved to activate ATF4, a 
transcription factor associated with cAMP-dependent renal disease in JS patients [15]. Up to now, dozens of variants in CEP290 have been identified to cause JBTS5 (OMIM\#610188) [16], a subtype of JS mainly associated with serve retinal and renal involvement in affected individuals [17]. However, almost all variants resulted in premature stop codon in CEP290 mRNA and truncated, nonfunctional CEP290 protein due to nonsense or frameshift variants, and the most frequent in which was c.5668G $>\mathrm{T}$ [p.G1890*] $[15,18,19]$. Interestingly, only one research reported a deletion from intron 43 of CEP290 to C12orf29 (chromosome 12 open reading frame 19) [20]; there were no other CNVs in CEP290 were reported in JS patients yet.

In this study, we presented clinical and molecular findings from a family with two JS affected siblings. Both of them carried a novel nonsense variant and a novel deletion in CEP290 inherited from their parents respectively. The pathogenesis is compound heterozygous variants lead to biallelic loss-of-function of CEP290, which has been associated with JS clearly.

\section{Results}

\section{Clinical summary}

An unrelated natural couple brought a 4-year-old boy with delayed development to the outpatient for genetic counseling (Fig. 1a). The proband exhibited features of moderate vision impairment, disorders of intellectual development, biparietal narrowing, and ataxia mainly on abnormal oculomotor (nystagmus) (Fig. 1b). Remarkably, the proband had a 10-year-old brother sharing the similar phenotype on vision impairment, intellectual development and oculomotor, and specially presenting gait disturbance (gait-limb incoordination) and abnormal form of the vertebral bodies (Fig. 1c). The axial brain magnetic resonance imaging (MRI) was then performed on these two siblings. Strikingly, hypoplastic cerebellar vermis, abnormal fourth ventricle, elongated superior cerebellar peduncles, and so-called "molar tooth sign" (MTS) were found on both of the two affected individuals (Fig. 1d, e). There was no functional and morphological abnormality of their kidney, lung, and liver so far. Thus, we initially diagnosed these two siblings with JS depending on the specific clinical characteristics. There was no phenotypic abnormality discovered in their parents.

\section{Molecular genetic analysis}

To elucidate the underlying genetic cause of JS in this family, we performed WES on all the family members. Importantly, a novel heterozygous nonsense variant a
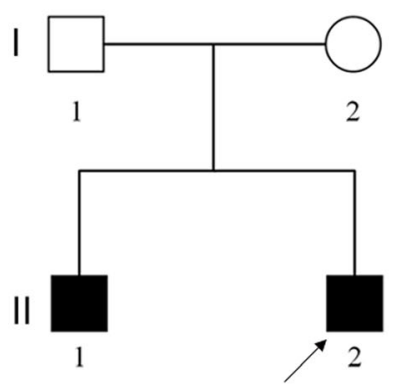

d

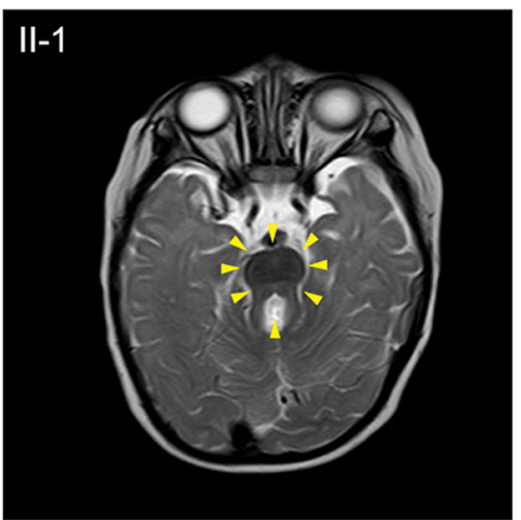

b

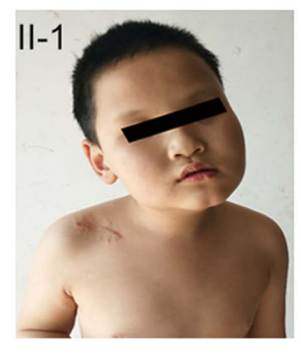

C

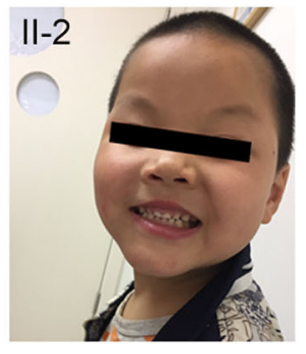

e

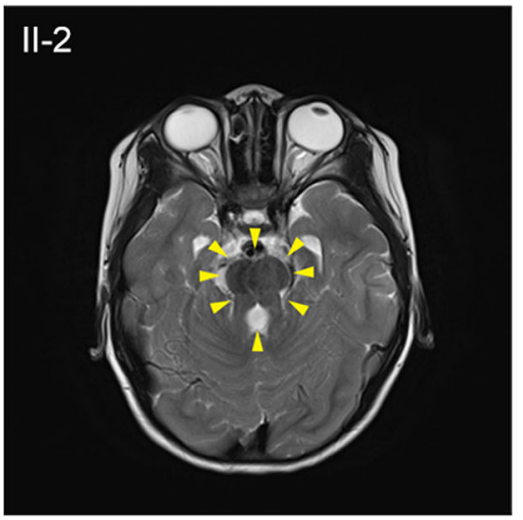

Fig. 1 Clinical summary for a JS family. a Family pedigree. An unrelated natural couple who gave birth to two affected siblings (black arrow denotes the proband). $\mathbf{b}$-e The affected siblings and their axial brain MRI (Yellow arrowheads showed the thick and long superior cerebellar peduncles, forming the roots of the so-called "molar tooth sign") 
(c.5953G>T [p.E1985*]) in CEP290 gene, a known causative gene of JS, was identified in the proband, his affected brother, and his mother but not the father. Furthermore, there was no report about this variation in any database, including ExAC browser, 1000 genome project, or GnomAD. In addition, this variant site is $100 \%$ highly conserved in many species (Fig. 2a). However, the nature of JS associated with CEP290 is an autosomal recessive inheritance, so we considered that there should be another variant in CEP290. We further analyzed the WES data, and strikingly, a suspected $65.97-\mathrm{kb}$ deletion in 12q21.32 (chr12, 88523465-88589431) was found in the two affected individuals and their father but absent in the mother (Fig. 2b), which covered exons 1 to 10 of CEP290. Furthermore, this CEP290 deletion was confirmed on the proband by chromosome CGH analysis. As expected, a heterozygous 298.1-kb deletion in 12q21.32 (chr12, 88525732-88823847) was detected out, which gives rise to exon 1 to exon 6 deletion of CEP290 (Fig. 2c). In view of the coding sequence (CDS) region of CEP290 started in exon 2, the deletion detected in this family causes a complete translation deficiency. These findings concluded that the biallelic loss-of-function of CEP290 variations was the genetic cause of JS in this family.

\section{The identification and negative effects of CEP290 variants in the JS family}

To understand the putative contribution of the singlenucleotide variant (c.5953G $>\mathrm{T}$ [p.E1985*]) to the proband's phenotype, we performed Sanger sequencing on this family, and this variant was observed in the proband, his affected brother, and their mother, meanwhile which was absent in their father (Fig. 3a). This variation resulted in a premature stop codon in transcribed mRNA (NM_025114.3, exon 43) and thus a truncated CEP290 protein, which leads to the missing of $\mathrm{CC}$ (coiled-coils) domain XIII, P-loop (ATP/GTP-binding site motif A) domain, KID (RepA/Rep+ protein kinase interaction domain) IV-VI(15) resulting in the loss-offunction of CEP290 protein (Fig. 3b).

To verify the $\mathrm{CNV}$, qPCR was carried out to analyze genome relative copy number of exon 1 , exon 2 , exon 6 , exon 7, exon 10, and exon 11 of CEP290 in the family. Results showed that compared to the mother, the father and siblings only had relative half-fold copy for exon 1 , exon 2 , exon 6 , exon 7 , and exon 10 , meanwhile exon 11 were equivalent (Fig. 3c). Furthermore, leukocyte mRNA levels of CEP290 also revealed significant decrease in the two siblings compared to their parents by qPCR (Fig. $3 d)$. Thus, these novel biallelic loss-of-function variants in CEP290 could significantly reduce CEP290 expression and further caused JS in these two siblings.

\section{Discussion}

In this study, a novel genetic cause associated with a heterozygous nonsense variant combined a heterozygous deletion of a large fragment within CEP290 was identified in two JS patients, which uncovered a novel pathogenesis of JS and provide more clue for genetic diagnosis of this disease

To date, variants of CEP290 have been demonstrated with several genetic disorders more than JBST5, such as Leber congenital amaurosis (LCA10, OMIM\#611755) [17], Meckel syndrome type 4 (MKS4, OMIM\#611134) $[21,22]$, and Bardet-Biedl syndrome 14 (BBS14, OMIM\#615991) [23, 24]. It is wondering that why variations in one gene could result in numbers of different syndromes. In 2010, Coppieters et al. reviewed over 100 distinct variants in CEP290 and indicated that no clear genotype-phenotype correlation could be established and the reason may be insufficient understanding of second-site modifiers alleles, such as variants in other genes encoding ciliary protein and interact with CEP290 [10]. In addition, we also hold the point that different variant subtypes in this gene may cause specific syndromes, as there are so many different domains with different vital functions in this large protein [14, 15]. In this study, we found two novel variants of CEP290, which may help to understand mechanisms of variation in CEP290.

Currently, WES has been frequently used and is an effective scheme for genetic disorder diagnosis, especially rare or non-fully clear diseases. However, some CNVs, including deletions and duplications, cannot be readily detected by WES [25]. Thus so, missing detecting CNV may give rise to unexplained genotype-phenotype uncorrelation in some disease. In our study, initially, in view of the proband shared the typical JBTS5 phenotype, but their parents claimed just a heterozygous CEP290 nonsense variant was found in prior genetic diagnosis (details unknown). We therefore performed WES and chromosome $\mathrm{CGH}$ to focus on both $\mathrm{SNV}$ and $\mathrm{CNV}$ in CEP290. Expectedly, nonsense variant and deletion were detected out in the two patients. Our investigation of JS in this family showed it was very essential to use combined methods to study pathogenesis of genetic disorders.

\section{Conclusions}

In conclusion, in this study, we unveiled two new variants, a SNV and a CNV of CEP290, which caused a complete translation deficiency of CEP290 leading to JS in two siblings. Specially, such deletion over $100 \mathrm{~kb}$ in CEP290 detected in JS individuals was first reported. These findings may help to conclude and reveal specific variation types in CEP290, which is benefit for the diagnosis and then for the future precision treatment of JS. 
a

H.sapiens

D.rerio

M.musculus

D.melanogaster

M.mulatta

Conservation

Quality

Consensus

LKT T GMT V D QV L GV RALES EKELEELKKRNL D L E LKT T GMTV DQVLGVRALES EKELEELKKRNLDLE LKT T GMTV DQVLG I RALES EKELEELKKRNLDLE LKT T GMTV D QVL GVRALESEKELEELKKKNL D L E LKGRGVTADQVVGART LEADKE I E L HKRNAELE
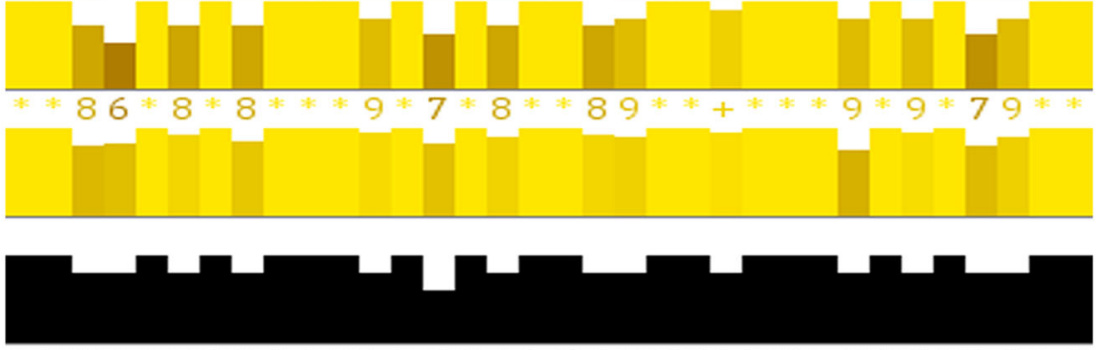

LKT T GMTV DQVLGVRALESEKELEELKKRNL D LE

b

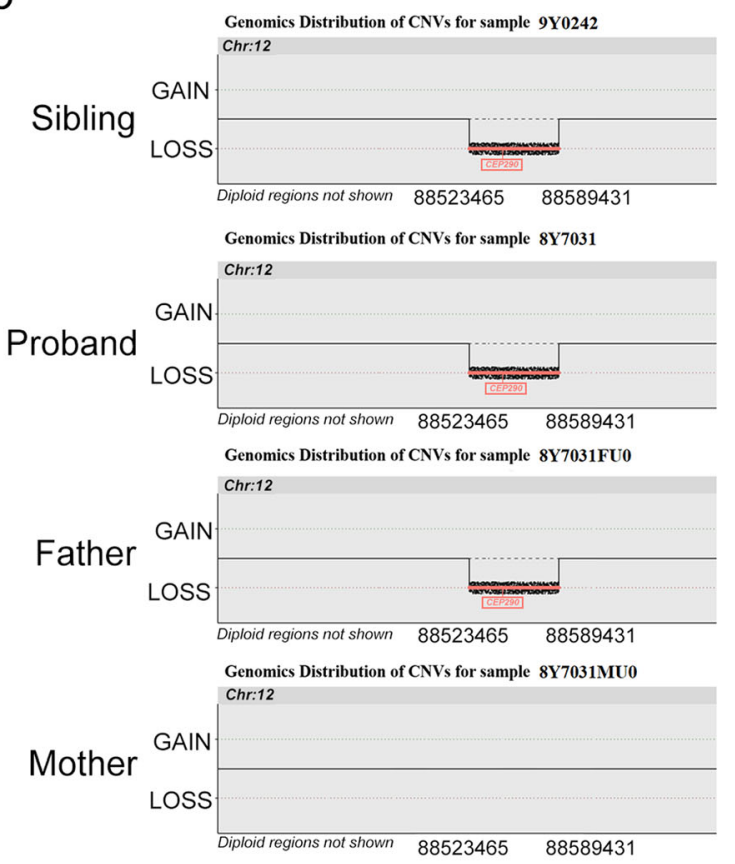

C

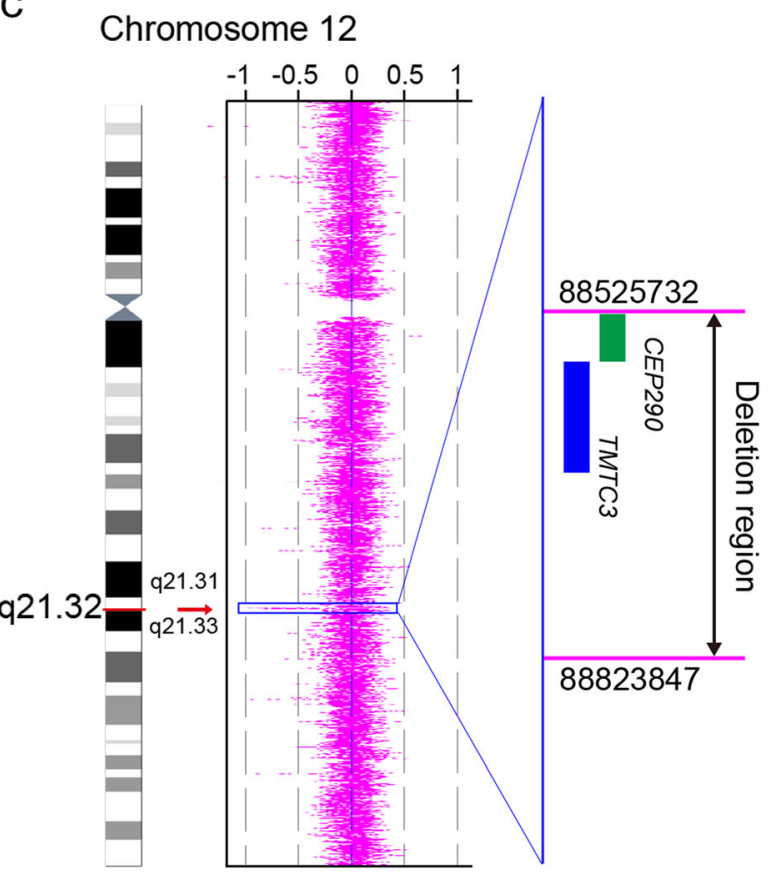

Fig. 2 Variants identified from WES and chromosome CGH. a Multiple sequence alignment of the CEP290 protein for different species. (black arrow denotes the position of the variant) (c.5953G>T [p.E1985*]). b WES identified a suspected 65.97-kb deletion in 12q21.32 which affected CEP290 in the father and siblings (8Y7031: proband, 9Y0242: sibling, 8Y7031FU0: father, 8Y7031MU0: mother). c Chromosome CGH confirmed a 298.1-kb deletion in 12q21.32 which affected CEP290 in the family

\section{Methods}

\section{Subjects}

Peripheral blood samples were obtained from the proband and his family after informed consent was signed respectively. Experiment on human subjects was approved by the Ethical Review Board of West China Second University Hospital, Sichuan University. The two affected siblings' brain MRI results were evaluated by two pediatric neuroradiologists.

Whole-exome sequencing and chromosome comparative genomic hybridization

Genomic DNA was extracted from peripheral blood leukocytes using whole blood DNA purification kit 


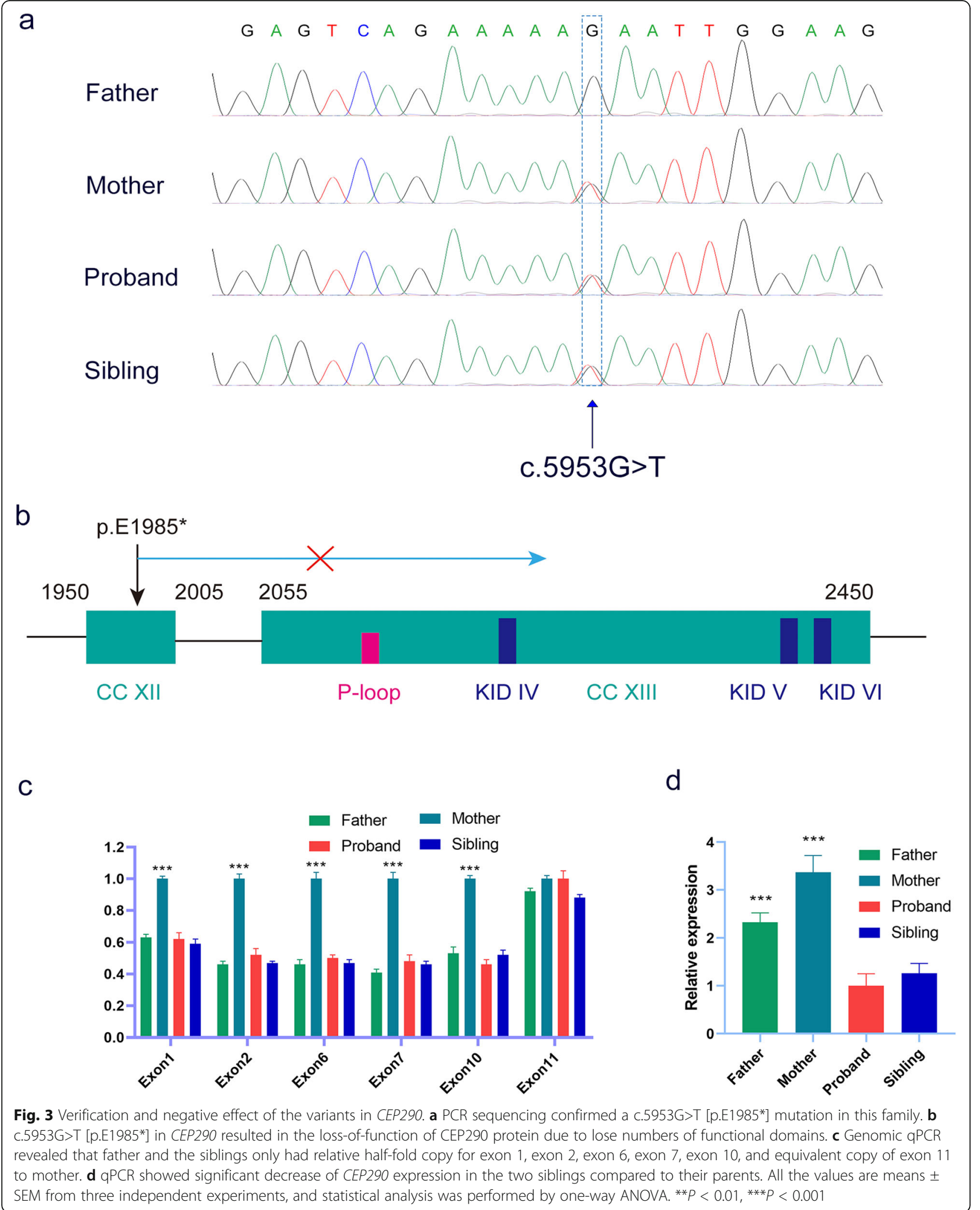


Table 1 Primers used in the current study

\begin{tabular}{|c|c|c|}
\hline Primers & Forward & Reversed \\
\hline CEP290 SNV & 5' TAAATTCCACAGAGCCGATAAA 3' & 5' ACAGCCCAAGAAATGAGGTT 3' \\
\hline CEP290 exon 1 & 5' GTTCCACGCCTTCTCATCAT 3' & 5' TGCCAGGAGAGCCTACAGTT 3' \\
\hline CEP290 exon 2 & 5' AGGTGGAGCACAGTGAAAGAA 3' & 5' TCTGCCAGTTCTTCTTGACG 3' \\
\hline CEP290 exon 6 & 5' AGTCTGCAGGTGGACGAGAT 3' & 5' CCAACTCCTITTCCATGTCC 3' \\
\hline CEP290 exon 7 & 5' CAGCCTAGGCGACAAAGACT 3' & 5' CCTITGTTGAACCACCACAA 3' \\
\hline CEP290 exon 10 & 5' GGACACTTATGGCTGCGTTT 3' & 5' CATCAGTCATCTTCTCCATTTCC 3' \\
\hline CEP290 exon 11 & 5' CATCAGTTTGCAACAACTCTTGA 3' & 5' TITTGCATTGACAGCTACCAT 3' \\
\hline CEP290 mRNA & 5' AAAGTTGACCCAGATGACCT 3' & 5' AAACCGAGTATCTCGTCCAC 3' \\
\hline Human GAPDH & 5' ATGTTCGTCATGGGTGTGAA 3' & 5' GTCTTCTGGGTGGCAGTGAT 3' \\
\hline
\end{tabular}

(TIANGENE). For WES, exons were captured from $1 \mu \mathrm{g}$ genomic DNA using Agilent SureSelect Human All Exon V6 Kit and then sequenced on Illumina HiSeq X system under instructions provided by the manufacturer. Functional annotation was performed through ANNOVAR and data were filtered by public database, such as ExAC, 1000 Genomes Project, and HGMD.

For chromosome comparative genomic hybridization, genomic DNA was digested with Nsp1 and ligated to adaptors. PCR amplicon was performed using primer pairs provided by the manufacturer, and the products were then purified and digested with DNase I to produce varying length DNA fragments. The fragments were labeled with biotinylated base and then hybridized with a pre-equilibrated Affymetrix chip Cytoscan HD chip. The chip consists of 2,670,000 probes that include $1,950,000$ non-polymorphic probes for analysis CNVs. After washing and staining, the chip was scanned with the GeneChip Scanner and the signal intensity for each marker is assessed. The results were analyzed using the Chromosome Analysis Suite (version 3.3) with regard to aberrations minimally sizing $100 \mathrm{~kb}$ and according to public database including Database of Genomic Variants (DGV), Database of Chromosomal Imbalance and Phenotype in Humans using Ensembl Resources (DECIPHER), and UCSC human genome build 19. The analysis results were showed as $\log 2$ ratio of the intensities and allele peak distribution.

\section{Genomic DNA sequencing and quantitative PCR}

Genomic DNA was obtained from peripheral blood leukocytes using whole blood DNA purification kit (TIANGENE). PCR amplification was performed using primer pairs (Table 1) designed to cover variant identified by WES. Sequencing of PCR products was conducted on an ABI377A DNA sequencer (Applied Biosystems). Data were evaluated using the Chromas software.

Total RNA was extracted using TRIzol reagent (Invitrogen, 15596026), and then reversed to cDNA through RT reagent Kit (Takara, RR037A). Triplicate quantitative
PCR for genomic DNA or cDNA was performed using SYBR Green qPCR Master Mix (Bimake, B21202) on an iCycler RT-PCR Detection System (Bio-Rad Laboratories). Delta-delta CT value analysis method was used to evaluate genome exon 1 , exon 2 , exon 6 , exon 7 , exon 10 , and exon 11 relative copy number, or exon 3 to 5 mRNA expression for all samples. Target and internal control gene primer pairs were listed below (Table 1).

\section{Abbreviations \\ JS: Joubert Syndrome; MRI: Magnetic resonance imaging; MTS: Molar tooth sign; JSRD: Joubert syndrome-related disorders; CNV: Copy number variation; WES: Whole-exome sequencing; $\mathrm{CGH}$ : Comparative genomic hybridization; SNV: Single-nucleotide variants \\ Acknowledgements \\ We thank the patient and his family for their interest and cooperation.}

\section{Authors' contributions}

Ying Shen designed the study experiments. Zhu Zhang collected the data and conducted the clinical evaluations. Xueguang Zhang and Xiang Wang performed PCR-seq and qPCR. Xiang Wang wrote the article. Hongqian Liu supervised the study experiments. All authors revised and approved the article.

\section{Funding}

This work was supported by General Program of China Post-doctoral Science Foundation (2018 M640920) and the Research Project of Science and Technology Bureau of Chengdu (No. 2016-HM01-00257-SF).

\section{Availability of data and materials}

The data that support the findings of this study are available from the corresponding author upon reasonable request.

Ethics approval and consent to participate

Experiment on human subjects was approved by the Ethical Review Board of West China Second University Hospital, Sichuan University.

Informed consent for participation to this study was obtained from all individuals.

\section{Consent for publication}

Publication of data was informed consent to all individuals involved in this study.

\section{Competing interests}

The authors declare no conflict of interests.

\section{Author details}

${ }^{1}$ Department of Obstetrics/Gynecology, Joint Laboratory of Reproductive Medicine (SCU-CUHK), Key Laboratory of Obstetric, Gynecologic and Pediatric 
Diseases and Birth Defects of Ministry of Education, West China Second University Hospital, Sichuan University, Chengdu 610041, China. ${ }^{2}$ Department of Obstetrics and Gynecology, West China Second University Hospital, Sichuan University, Chengdu 610041, China. ${ }^{3}$ Key Laboratory of Birth Defects and Related Diseases of Women and Children, Ministry of Education, Sichuan University, Chengdu 610041, China.

Received: 9 January 2020 Accepted: 11 June 2020

Published online: 29 June 2020

\section{References}

1. Joubert M, Eisenring JJ, Robb JP, Andermann F. Familial agenesis of the cerebellar vermis. A syndrome of episodic hyperpnea, abnormal eye movements, ataxia, and retardation. Neurology. 1969;19(9):813-25.

2. Boltshauser $\mathrm{E}$, Isler W. Joubert syndrome: episodic hyperpnea, abnormal eye movements, retardation and ataxia, associated with dysplasia of the cerebellar vermis. Neuropadiatrie. 1977;8(1):57-66.

3. Wang SF, Kowal TJ, Ning K, Koo EB, Wu AY, Mahajan VB, et al. Review of ocular manifestations of Joubert syndrome. Genes (Basel). 2018;9(12).

4. Gleeson JG, Keeler LC, Parisi MA, Marsh SE, Chance PF, Glass IA, et al. Molar tooth sign of the midbrain-hindbrain junction: occurrence in multiple distinct syndromes. Am J Med Genet A. 2004;125A(2):125-34 discussion 17.

5. Maria BL, Hoang KB, Tusa RJ, Mancuso AA, Hamed LM, Quisling RG, et al. "Joubert syndrome" revisited: key ocular motor signs with magnetic resonance imaging correlation. J Child Neurol. 1997;12(7):423-30.

6. Brancati F, Dallapiccola B, Valente EM. Joubert Syndrome and related disorders. Orphanet J Rare Dis. 2010;5:20.

7. Romani M, Micalizzi A, Valente EM. Joubert syndrome: congenital cerebellar ataxia with the molar tooth. Lancet Neurol. 2013;12(9):894-905.

8. Doherty D. Joubert syndrome: insights into brain development, cilium biology, and complex disease. Semin Pediatr Neurol. 2009;16(3):143-54.

9. Friede RL. Developmental neuropathology: Springer Science \& Business Media; 2012

10. Coppieters F, Lefever S, Leroy BP, De Baere E. CEP290, a gene with many faces: mutation overview and presentation of CEP290base. Hum Mutat. 2010;31(10):1097-108.

11. Badano $\mathrm{J}$, Teslovich $\mathrm{TM}$, Katsanis $\mathrm{N}$. The centrosome in human genetic disease. Nat Rev Genet. 2005;6(3):194-205.

12. Doxsey S. Re-evaluating centrosome function. Nat Rev Mol Cell Biol. 2001; 2(9):688-98.

13. Bachmann-Gagescu R, Dempsey JC, Phelps IG, O'Roak BJ, Knutzen DM, Rue TC, et al. Joubert syndrome: a model for untangling recessive disorders with extreme genetic heterogeneity. J Med Genet. 2015;52(8):514-22.

14. Drivas TG, Holzbaur EL, Bennett J. Disruption of CEP290 microtubule/ membrane-binding domains causes retinal degeneration. J Clin Invest. 2013; 123(10):4525-39.

15. Sayer JA, Otto EA, O'Toole JF, Nurnberg G, Kennedy MA, Becker C, et al. The centrosomal protein nephrocystin-6 is mutated in Joubert syndrome and activates transcription factor ATF4. Nat Genet. 2006;38(6):674-81.

16. Valente EM, Silhavy JL, Brancati F, Barrano G, Krishnaswami SR, Castori M, et al. Mutations in CEP290, which encodes a centrosomal protein, cause pleiotropic forms of Joubert syndrome. Nat Genet. 2006;38(6):623-5.

17. den Hollander Al, Koenekoop RK, Yzer S, Lopez I, Arends ML, Voesenek KE, et al. Mutations in the CEP290 (NPHP6) gene are a frequent cause of Leber congenital amaurosis. Am J Hum Genet. 2006;79(3):556-61.

18. Brooks BP, Zein WM, Thompson AH, Mokhtarzadeh M, Doherty DA, Parisi M, et al. Joubert syndrome: ophthalmological findings in correlation with genotype and hepatorenal disease in 99 patients prospectively evaluated at a single center. Ophthalmology. 2018:125(12):1937-52.

19. Ben-Salem S, Al-Shamsi AM, Gleeson JG, Ali BR, Al-Gazali L. Mutation spectrum of Joubert syndrome and related disorders among Arabs. Hum Genome Var. 2014;1:14020.

20. Travaglini L, Brancati F, Attie-Bitach T, Audollent S, Bertini E, Kaplan J, et al. Expanding CEP290 mutational spectrum in ciliopathies. Am J Med Genet A. 2009;149A(10):2173-80

21. Baala L, Audollent S, Martinovic J, Ozilou C, Babron MC, Sivanandamoorthy S, et al. Pleiotropic effects of CEP290 (NPHP6) mutations extend to Meckel syndrome. Am J Hum Genet. 2007;81(1):170-9.

22. Molin A, Benoist G, Jeanne-Pasquier C, Elkartoufi N, Litzer J, Decamp M, et al. 12q21 Microdeletion in a fetus with Meckel syndrome involving CEP290/MKS4. Eur J Med Genet. 2013;56(10):580-3.
23. Li JB, Gerdes JM, Haycraft CJ, Fan Y, Teslovich TM, May-Simera H, et al. Comparative genomics identifies a flagellar and basal body proteome that includes the BBS5 human disease gene. Cell. 2004;117(4):541-52.

24. Slavotinek A, Beales P. Clinical utility gene card for: Bardet-Biedl syndrome. Eur J Hum Genet. 2011;19(3):3-4.

25. de Ligt J, Boone PM, Pfundt R, Vissers LE, Richmond T, Geoghegan J, et al. Detection of clinically relevant copy number variants with whole-exome sequencing. Hum Mutat. 2013;34(10):1439-48.

\section{Publisher's Note}

Springer Nature remains neutral with regard to jurisdictional claims in published maps and institutional affiliations.
Ready to submit your research? Choose BMC and benefit from:

- fast, convenient online submission

- thorough peer review by experienced researchers in your field

- rapid publication on acceptance

- support for research data, including large and complex data types

- gold Open Access which fosters wider collaboration and increased citations

- maximum visibility for your research: over $100 \mathrm{M}$ website views per year

At $\mathrm{BMC}$, research is always in progress.

Learn more biomedcentral.com/submissions 